\title{
Being Positive About Negative Facts
}

\author{
STEPHEN BARKER \\ MARK JAGO Philosophy \& Phenomenological Research, forthcoming
}

\begin{abstract}
Negative facts get a bad press. One reason for this is that it is not clear what negative facts are. We provide a theory of negative facts on which they are no stranger than positive atomic facts. We show that none of the usual arguments hold water against this account. Negative facts exist in the usual sense of existence and conform to an acceptable Eleatic principle. Furthermore, there are good reasons to want them around, including their roles in causation, chance-making and truth-making, and in constituting holes and edges.
\end{abstract}

\section{Introduction}

7 Here Is No HipPo in the Nottingham University Lake. This fact becomes evident to anyone who spends time by the lake and knows something about the habits of hippos and lake life. It is one among many other facts about the lake that become evident through cursory inspection. Part of the reason why swimming in the lake is safe is the fact that there are no hippos in the lake. The thing that explains why that swimmer made it across just then-the guy in the pink trunks-whereas that swimmer on the Zambezi didn't make it across that river, is the fact that our lake lacks a hippo keen to devour swimmers. And so it goes. Our little disquisition about aspects of our favourite lake made free use of talk of facts, including negative facts. We quantified over these facts and explained a few things by invoking them. Nothing could be more unproblematic. Talk of negative facts is banal and no stranger than talk of any other facts. Yet there is a minor philosophical industry that has cast negative facts in the light of dubious dark entities to be purged from all respectable inventories of the world. We think the would-be purgers are misguided; indeed, they lack any cogent rationale for their enmity to the negative. The negative is just part of ordinary ontological commitment. There is no reason to think that this commitment is defective and many reasons to think it is right.

What drives this enmity to negative facts? We think it is the vampiric image of negative facts as amongst the metaphysically undead: non-existence masquerading as existence. We look at such arguments in $\mathbb{\$} 2$ and, in $\mathbb{S} \mathbb{S}_{3}-5$, develop an account of negative facts that puts these worries to rest. But there are further worries with negative facts, involving various forms of the Eleatic principle. We address worries that negative facts are causally inert $(\$ 6)$, that they cannot constitute material objects $(\mathbb{7} 7)$ and that they cannot be perceived $(\mathbb{8})$. Finally, in $\$ 9$, we show why negative facts are good philosophical friends to have around. 


\section{Some Initial Worries About Negative Facts}

It is common to paint a vampiric image of negative facts. In this section, we survey some of the immediate objections to negative facts which motivate this macabre conception of them. ${ }^{\mathrm{I}}$ Although these arguments do not stand up to closer inspection, they do establish desiderata for the theory of negative facts in $\mathbb{S}_{3}$. Mumford (2007) is someone keen to persuade us of the non-existence of negative facts. He says that

A fact ... is taken to be some kind of existent in the world.... But ... can it really be a fact in the world that there is no hippopotamus in the room? This sounds like an absence of a fact, and an absence is nothing at all. $(2007,46)$

We take Mumford's point to be that a negative fact would have to be an absence, but an absence is nothing at all, i.e., a non-existent object. But of course, we want our negative facts to exist just as much as our positive facts. So our first desideratum is:

(I) The theory must show how negative facts qua absences can have any existence at all.

A related worry, pushed by Molnar (2000, 84-5) and others, is that everything that exists is positive. But negative facts are not positive; and again, it looks like they are debarred from the realm of being. We have some worries about exactly what Molnar and followers might mean by 'positive': we see what it would be for a representation (such as a sentence) to be positive or negative, but facts are not representational entities. ${ }^{2}$ Still, we take Molnar's point that there is but one notion of existence (and we shan't enter into whether it should be called 'positive'). So our second desideratum is:

(2) The theory must show how negative facts exist in just the same sense of 'existence' as everything else (including positive facts).

With these two desiderata in mind, we now proceed to give our theory of negative facts.

\section{Negative Facts Resurrected}

We will present our theory of facts over the next three sections. In this section, we give the philosophical basis for the theory. In $\$ 4$, we further elaborate the theory by comparing it to others on the market. Then, in $\$ 5$, we set out the formal details

I. There also exist arguments against facts in general such as the infamous slingshot argument, which purports to show that there is only one fact (see, e.g., Neale 200I). We think that the slingshot is over-rated, as do many others (see Restall 2004).

2. Parsons $(2006,59 \mathrm{I}-2)$ expresses doubts about the coherence of describing entities as 'positive' or 'negative'. 
of the theory and use it to analyse conjunctive, negative existential and universal facts.

Let's begin with (what we take to be) the established theory of facts as substantial metaphysical entities, due to Armstrong (1997). Consider the state of affairs the lake's being frozen. On Armstrong's view, this state of affairs consists of the 'thin' particular, the lake, possessing the property of frozenness. We denote that complex '[frozenness lake]'. Both the thin particular and the property are constituents of the complex. But the complex cannot be the mereological fusion of lake and property, for the lake and the property frozenness (and thus their mereological fusion) exist, yet the lake is not at present frozen and hence the fact the lake's being frozen does not exist. Nor can that fact be the mereological fusion of lake, property and some other relation $\mathrm{R}$ which ties particular and property together. For suppose there is such a relation $\mathrm{R}$ : then lake, property and relation $\mathrm{R}$ all exist, hence so does their mereological fusion, yet the complex [frozenness lake] does not. Whatever supposed constituents we throw into the mereological mix, it will never suffice for the fact in question.

What, then, is the unity of the complex [frozenness lake]? Armstrong's answer is that it is an irreducible non-mereological unity. Facts are irreducible nonmereological wholes in that they have constituents but they are something over and above those constituents. ${ }^{3}$ On Armstrong's view, moreover, facts (states of affairs) are the fundamental entities, whereas properties and relations are 'states of affairs types' (I997, I 27). Those properties and relations are abstractions from (and thus ontologically derivative upon) particular facts. ${ }^{4}$ Is non-mereological composition too much to believe? Any theorist taking seriously the ontological grounds of predication must embrace ideas of comparable strangeness. ${ }^{5}$ We say: choose your poison and see what work you can do with it. As we show below, non-mereological composition in the guise of facts does a lot of work.

We accept Armstrong's story but add to it this: non-mereological wholes come in a variety of flavours. If the lake's being frozen is the state of affairs that results when the (thin particular) lake is tied to the property of frozenness in one way, then the lake's not being frozen is the state of affairs that results when the (thin particular) lake is tied to the property of frozenness in another way. If the first way is instantiation, then the second is anti-instantiation. ${ }^{6}$ The first way gives one kind of non-mereological whole, the second gives another. Neither kind of tie nor corresponding kind of whole is reducible to the other kind of tie or whole.

As neither form of the ontological tie is a relation between particulars and

3. Some are tempted to talk of 'non-mereological ties' linking constituents. Instantiation is meant to be such a link. We are not averse to that mode of talk, but we must emphasise that instantiation cannot be an extra constituent of the fact. Rather, saying that lake and property are linked by a non-mereological tie is just a fancy way of saying that they are parts of a non-mereological fusion of a certain kind.

4. A 'state of affairs type' is an abstraction from a class of particular facts, 'unified by the universal [i.e., the property or relation] that they contain' (I997, I 27).

5. Lewis (I983), for example, rejects non-mereological composition but must accept primitive instantiation for the basic relation of set-membership that underpins his analysis of predication.

6. Again, we must emphasise that neither kind of non-mereological tie (neither instantiation nor anti-instantiation) is a constituent of any kind of fact. 
properties or relations (and so neither tie is a constituent of any state of affairs), we will continue to use the notation '[Fa]' for facts whose non-mereological constituents are the individual $a$ and the property $F$. To differentiate between the kinds of tie involved, we will write ' $[\mathrm{Fa}]^{+}$' for the state of affairs of a's being $\mathrm{F}$ and ' $[\mathrm{Fa}]^{- \text {' }}$ for a's not being $\mathrm{F}$. More generally, if $\mathrm{R}$ is a primitive relation which holds between $n$ particulars and $O_{1}$ to $O_{n}$ are particulars, then $\left[R o_{1} \cdots O_{n}\right]^{+}$is a positive atomic state of affairs and $\left[\mathrm{Ro}_{1} \cdots \mathrm{O}_{\mathrm{n}}\right]^{-}$is a negative atomic state of affairs.

We view facts, including negatives facts, as spatiotemporally located entities. Their spatiotemporal location is given by the spatiotemporal location of their concrete constituents. The fact that Fred is happy at time $t$ is spatially located where Fred is at $t$ and temporally located at $t$. A positive relational fact is located where and when its relata are located insofar as the relation is instantiated by them. The location of the fact that Uluru is 25 kilometres from Kata Tjuta is a discontinuous region, comprising the regions occupied by Uluru and Kata Tjuta. Likewise for negative facts: the fact that the Eiffel Tower is not next to the Sydney Opera House is located at the discontinuous region occupied by the Eiffel Tower and the Sydney Opera house.

Talk of absences, lacks and the ways things aren't picks out negative facts, just as talk of the ways things are picks out positive facts. When one says 'the absence of a hippo in the lake', one denotes the negative fact that there is no hippo in the lake (We discuss negative existential facts in $\$ 5$.) This picture of negative facts (and of absences and lacks) meets the desiderata from $\$ 2$. There is a tendency, in using absence-talk, to confuse that which is absent (lake-dwelling hippos, say) with that which does exist (the absence itself). Absences can exist, on our picture, because they are identical to negative facts. There is no contradiction here: when we say that there is an absence of a hippo in the lake, it is lake-dwelling hippos that do not exist. The fact that there is no hippo in the lake exists. That fact is not a lake-dwelling hippo and so we are not claiming that something both exists and does not exist. So desideratum (I) is met.

Secondly, Molnar's worry about positing a negative form of existence is rebutted by our theory: negative facts exist in just the same sense of 'existence' as positive facts (and every other kind of being). Negative facts are non-mereological wholes just as positive facts are and so have the same kind of existence. What differs between negative and positive facts is the kind of non-mereological composition involved. So desideratum (2) is met. In addition, negative facts contain the same kind of constituents as positive facts: hippos, lakes, the property of frozenness and the like. So, if one accepts our positive facts, one cannot then object to our negative facts on the grounds that they contain dubious 'negative' particulars. ${ }^{7}$

We should point out the distinction between allowing negative facts, as we do, and allowing negated facts, as others do (e.g., Fine (I982), Zalta (I993) and

7. Mumford $(2007,46)$, for example, worries that a negative fact that there is no hippopotamus in the room might be taken to involve a highly dubious 'non-hippopotamus' as a constituent. 'What, though', Mumford rhetorically asks, 'is a non-hippopotamus and where exactly in the room is it?' $(2007,46)$. But we postulate no such entity (see $\$ 5$ on how we handle negative existential facts). 
Restall (2004)). ${ }^{8}$ On the latter views, negated facts have a complex structure similar to that of negated sentences. But this raises many difficult issues. Whilst a material spatiotemporally located fact may be perceived, referred to or ignored, we do not see how it is the kind of thing that can be negated. ${ }^{9}$ Facts, for us, are not abstract or theoretical entities. In contrast, we do not posit any negation operation on facts and so do not think of our negative facts as the negations of positive facts. Negation is nowhere represented in the structure of our negative facts and this, it seems to us, is an advantage of our view, for we want negative as well as positive facts to be in principle perceivable (see $\$ 8$ ).

\section{Comparison with Other Accounts}

Our theory (and the notation we use) invites comparison with Beall's (2000) and van Fraassen's (I969) theories of facts. According to Beall's theory, there exist polarities $\mathrm{O}$ and $\mathrm{I}$ and each atomic fact contains just one of these polarities. The fact that the lake is cold is of the form [coldness lake $\mathrm{I}$ ] whereas the fact that the lake is not frozen is of the form [frozenness lake o]. For Beall, positivity and negativity consist in having $\mathrm{I}$ and having $\mathrm{o}$, respectively, as a constituent. In a way, we also accept polarized facts but, importantly, ours are not polarized in virtue of any of their constituents. We do not think there is any reason to think that positive and negative facts differ in their constituents.

On Van Fraassen's account of facts, every property or relation $R$ has a dual $\bar{R}$, such that $O_{1}$ to $O_{n}$ are related by $\bar{R}$ iff they are not related by $R$. He then has the complex $\left[\bar{R} o_{1} \cdots o_{n}\right]$ where we have $\left[R o_{1} \cdots o_{n}\right]^{-} .{ }^{\text {Io }}$ But despite notational similarity, our theory is very different from van Fraassen's. His aim is to

explicate "fact" discourse in such a way that engaging in such discourse does not involve ontological commitment. This means that we must represent facts, relations among facts, and relations between facts and sentences ... without requiring the claim that it also represents a reality. (van Fraassen I969, 48I)

He goes on to construct set-theoretic representations of facts: atomic facts are ordered tuples, conjunctive facts are Cartesian products and universal facts are infinite conjunctions. ${ }^{\text {II }}$ On our theory, by contrast, (at least some) facts are concrete, spatiotemporally located entities. They are what it is for a given particular to have a given property, not merely a representation of it, and they are what grounds the truth of the corresponding predications.

8. Zalta speaks of states of affairs, rather then facts.

9. In Fine's and Zalta's theories, facts are language-like entities, whereas Restall takes facts to be sets of worlds. We understand how these kinds of entities can be negated but, for us, they are not facts: we take facts to be substantial entities, some of which are concrete and spatiotemporally located.

Iо. Van Frassen uses ' $(\cdot\rangle$ ' rather than '[.]' to denote complexes, for he identifies such complexes with ordered tuples (i.e., set-theoretic objects) (van Fraassen I969, 482).

II. Moreover, the properties and relations appearing in such complexes are themselves set-theoretic entities (they are the standard $\mathrm{n}$-ary Cartesian products on the domain used to interpret $\mathrm{n}$-place predicates in model theory). So a negative $n$-ary property $\bar{R}$ is just the complement of $R$ with respect to a domain $\mathfrak{D}$, i.e. $\mathfrak{D}^{\mathrm{n}}-\mathrm{R}$. 
Although we are at odds with van Fraassen's approach, this discussion has raised an interesting question. What if one accepted negative properties and relations (one for each positive property and relation), as in van Fraassen's theory, whilst treating facts as substantial parts of one's ontology, as we do? Then, in place of a negative fact $\left[R o_{1} \cdots o_{n}\right]^{-}$, one would have the positive fact $\left[\bar{R} o_{1} \cdots o_{n}\right]^{+}$. One could then get by without any negative facts. Is this account in competition with ours?

In one sense, the accounts are not in competition. We accept, along with Armstrong, that whatever property or relation can be abstracted from an existing fact is a genuine entity in its own right. As a consequence, we accept negative properties and relations into our ontology, as abstractions from negative facts. If $[\mathrm{Fa}]^{-}$is a negative fact, then $\lambda x[F x]^{-}$, the property of non-Fness which is abstracted from it, is a negative one. We then have a corresponding positive fact, $\left[\left(\lambda x[F x]^{-}\right) a\right]^{+}$, the fact that a possesses non-Fness. According to the theory we give in $\mathbb{S}_{5}$, this fact is identical to [Fa] ${ }^{-}$, for lacking Fness and possessing nonFness amount to the same thing. So, for all that has been said to far, the alternative account is no more than a notational variant of ours.

One way in which the alternative theory might differ from ours is if it takes negative properties to be fundamental entities. Suppose we take properties in general to be fundamental, and so think of the facts as containing those properties (in some ontological sense). Then, it seems to us, the question of how a fact contains a property has not been answered. This is just the question of the unity of facts, or of how particulars possess properties. Following Armstrong, by contrast, we take the facts to be fundamental and properties, as abstractions from facts, to be derivative entities. We posit two kinds of facts as basic entities and do not try to explain those entities further: rather, we want to see what work they can do within our theory, and assess their worth on that basis. This is why we deal primarily in terms of negative facts, rather than in terms of negative properties.

Here is a second reason for preferring a theory which deals primarily with negative facts, rather than negative properties. Properties (and property possession) are part of the explanandum. Our strategy is to postulate more fundamental kinds of entity-positive and negative facts—and account for properties (and property possession) in those terms. If we are justified in accepting fundamental positive and negative facts (and we argue below that we are), then we can account for positive and negative properties within our ontology. We can explain the explanandum. Alternatively, one could begin by positing positive and negative properties and then accounting for facts in those terms. But the existence and nature of facts is not part of the explanandum, for we should be pre-theoretically neural as to whether there are any substantial facts. So the strategy of beginning by positing negative properties appears to be at an explanatory disadvantage in comparison with our approach. 


\section{Conjunctive and Negative Existential Facts}

In this section, we give the remaining details of our theory of facts. First, we give the formal details; then, we show how to accommodate conjunctive, existential, negative existential and universal facts in the theory. ${ }^{\mathrm{I} 2}$ We base our theory on the typed $\lambda$-calculus. ${ }^{13}$ In our language for denoting facts, we have two base types: $f$, for fact-terms and $\mathrm{i}$, for constants denoting particulars. Types for predicates are constructed recursively from these: if $\sigma$ and $\tau$ are types and $\sigma \neq \mathrm{i}$, then $\tau \rightarrow \sigma$ is a type. For example, $i \rightarrow(i \rightarrow f)$ is the type of dyadic predicates of particulars.

We assume a stock of primitive predicates (which denote positive properties), constants and variables. Each of these comes with a fixed pre-determined type and each counts as a term. The remaining well-formed terms of our language are formed recursively as follows.

Application: Given terms 't' and ' $u$ ' of types $\tau \rightarrow f$ and $\tau$ respectively, '[tu] $]^{+}$, and ' $[t u]^{-}$' are well-formed terms, of type $f$. And given terms 't' and ' $u$ ' of types $\tau \rightarrow \sigma$ and $\tau$ respectively, where $\sigma \neq \mathrm{f}$, '(tu)' is a well-formed term, of type $\sigma$.

Abstraction: Given a term ' $t$ ' of type $\tau$ and a variable ' $x$ ' of type $\sigma$, ' $\lambda \mathbf{x}(\mathrm{t})$ ' is a well-formed term of type $\sigma \rightarrow \tau$.

For applications, we enclose all and only the terms of type $f$ in square brackets to highlight that these terms denote positive or negative facts (if they denote anything at all). All other complex terms denote properties of some kind (if they denote anything). ${ }^{\mathrm{I}}$ We give some examples at the end of this section.

We think of the syntactic operations of application and abstraction as corresponding to Armstrong's (I 997) ontological notions of non-mereological composition and the abstraction of properties and relations from facts $\left(\mathbb{3}_{3}\right)$. As we said above, we assume (along with Armstrong) that whatever property or relation can be abstracted from an existing fact is a genuine entity in its own right. Hence, when a term 't' denotes some fact and ' $\lambda x(t)$ ' can be abstracted (in the syntactic sense above) from ' $t$ ', we will assume that ' $\lambda x(t)$ ' also denotes a genuine property or relation. This will be important in our analysis of negative existential and universal facts, below. We will also assume that, for all terms ' $t$ ' and ' $u$ ' of types $\tau \rightarrow f$ and $f$ respectively, either the fact $[t u]^{+}$or the fact $[t u]^{-}$exists at any given world. ${ }^{15}$

I2. This section is based on Jago (20IOb), which gives more technical details of this approach. I3. There isn't space here to give an overview of the $\lambda$-calculus. We refer the interested reader to Barendregt I984; I992.

I4. Not every term in our language denotes an actual fact, property or relation (and some fact-terms may not even denote possible facts). Which facts exist at a given world (and hence which of our terms denote) is a contingent matter. Our theory is a theory of which kinds of fact are ontologically respectable, not of which particular facts exist.

I 5 . Having said that, nothing in what follows hangs on this classical principle. Indeed, interesting accounts can be given in terms of fact-gaps. For example, Sorensen (2004) and Greenough (2008) give theories of ontic vagueness is terms of fact-gaps whilst maintaining a classical approach to truth. 
In Jago (20Iob), a modified theory of reduction from the $\lambda$-calculus is used to provide identity conditions for facts. ${ }^{16}$ We adopt that theory here. The core of the theory is the following basic reduction-steps:

\begin{tabular}{|c|c|}
\hline$\left.\left.[\mathrm{Fx}]^{+}\right) \mathrm{a}\right]^{+}$ & reduces to \\
\hline$\left.\left.[F x]^{+}\right) a\right]^{-}$ & reduces to \\
\hline${ }^{\prime}\left[\left(\lambda \times[F x]^{-}\right) a\right]^{+}$ & reduces to \\
\hline${ }^{\prime}\left[\left(\lambda x[F x]^{-}\right) a\right]^{-}$ & reduces to \\
\hline
\end{tabular}

If ' $u$ ' is a sub-term of ' $t$ ' and ' $u$ ' reduces to ' $s$ ', then ' $t$ ' reduces to the term obtained by replacing ' $u$ ' with ' $s$ ' throughout ' $t$ '. The reduction relation is then the smallest preorder (reflexive and transitive relation) closed under these rules. We take reduction to entail identity: if term ' $t$ ' reduces to term ' $u$ ', then these terms denote the same entity (if they denote at all). The idea here is that the fact that a possesses the property of non-Fness is identical to the fact that a isn't F. ${ }^{17}$

Now we turn to our analysis of logically complex facts. Worries about conjunctive and disjunctive facts seem to us to stem from worries about the lack of conjunctive and disjunctive structure in the world. We agree that there are no worldly entities corresponding to the connectives 'and' and 'or', and so we do not think that there is a fact corresponding to every true sentence. What we can say, without ontological excess, is this. If there exist facts that $p$ and that $q$, then their mereological fusion exists (and exists only when those two facts exist). We don't see what's wrong with calling this entity the fact that $p \wedge q$. Where ' $\alpha$ ' and ' $\beta$ ' stand for facts, we denote their mereological fusion as ' $\alpha \oplus \beta$ ' and expand our definition of the well-formed terms accordingly. ${ }^{\mathrm{I} 8}$

As nothing stands to disjunction as the mereological fusion of two facts stands to conjunction, we do not accept disjunctive facts of any kind. In particular, if there is a possible fact that $p \wedge q$, there is no possible fact that $-(p \wedge q),{ }^{19}$ for this would be (equivalent to) a disjunctive fact, that $\neg p$ or $\neg q$. As a consequence, not every fact in our ontology has a complement. This is no problem for our theory. A fact has a complement when that fact is a non-mereological composition: the non-mereological fusions $[\mathrm{tu}]^{+}$and $[\mathrm{tu}]^{-}$are complements of one another. But conjunctive facts are mereological fusions of facts and so have no complements. ${ }^{20}$ Although we reject disjunctive facts, we still think that true disjunctions are made true by the facts. If the proposition $\langle p \vee q\rangle$ is true, then either $\langle p\rangle$ is true or $\langle q\rangle$ is true and so we can treat all those facts that make $\langle p\rangle$ true plus all those that make $\langle q\rangle$ true as the truthmakers for $\langle p \vee q\rangle$.

I6. Specifically, the theory comprises $\beta \eta$-reduction with extra clauses to handle the ' + ' and '-' superscripts on fact-terms.

I7. As a consequence, we can't assume that a fact is negative just because it is denoted by a term ending in a '-'. Since $[\mathrm{Fa}]^{+}$is a positive fact, $\left[\left(\lambda \times[F x]^{-}\right) a\right]^{-}$is too. They are one and the same fact, whose constituents are $\mathrm{F}$ and $\mathrm{a}$. What we can say is that a fact is positive (or negative) if it is denoted by a fully-reduced term ' $t$ ' which ends with a '+' (or '-').

I 8. We allow that ' $\alpha \oplus \beta$ ' is well-formed only when both ' $\alpha$ ' and ' $\beta$ ' are of type f. As ' $\alpha \oplus \beta$ ' is intended to denote a conjunctive fact, we assign it type $f$ too.

19. Except in the special case when ' $p$ ' = ' $q$ '.

20. This is why we write ' $\alpha \oplus \beta$ ' and not ' $[\alpha \oplus \beta]^{+}$'. If the latter was well-formed, then ' $[\alpha \oplus \beta]^{-}$' would be too, but we have no concept of what kind of entity such terms would denote. 
What about existential and universal facts? We don't think that there's any such thing as a property of existence to which we can appeal in our construction of existential facts. Nevertheless, there exist sensible higher-order properties of properties, such as the property of being instantiated, which will suffice for our needs. We introduce a primitive predicate 'In' to denote the property of

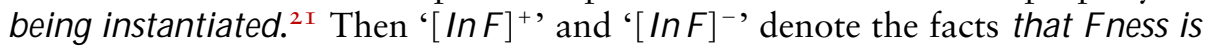
instantiated and that Fness isn't instantiated. If some entity $a$ is $F$ at $W$, then Fness possesses the property of being instantiated at $W$; but if there's no fact that $a$ is $F$ at $w$ for any $a$, then Fness lacks the property of being instantiated at $w$. So we can think of $[\mathrm{InF}]^{+}$and $[\mathrm{InF}]^{-}$and as existential and negative existential facts: the fact that something is $F$ and that nothing is $F$, respectively.

Now let's see what we can do with these facts in our ontology. The fact that the lake is large but not frozen is:

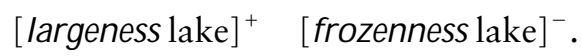

From this fact, we can abstract the property

$$
\lambda x\left([\text { largeness } \mathrm{x}]^{+} \oplus[\text { frozenness } \mathrm{x}]^{-}\right),
$$

the property of being largebut not frozen. We analyse the fact that all Fsare $G \mathrm{~s}$ as the fact that nothing has the property being an $F$ but not $a G, \lambda x\left([F x]^{+} \oplus[G x]^{-}\right)$. To say that nothing has this property, we say that the property is uninstantiated and hence we treat the fact that all Fs are GS as the negative fact

$$
\left[\ln \lambda x\left([F x]^{+} \oplus[G x]^{-}\right)\right]^{-} \text {. }
$$

We treat the fact that there is no hippo in the lake as the fact that nothing is both a hippo and contained by the lake, i.e.

$$
\left[\ln \lambda x\left([\text { hippo-hood } \mathrm{x}]^{+} \oplus[\text { containing lake } \mathrm{x}]^{+}\right)\right]^{-} \text {. }
$$

Similarly, the fact that there are no naturally purple hippos is the fact

$$
\left[\ln \lambda \times\left([\text { hippo-hood } \mathrm{x}]^{+} \oplus[\text { natural-purpleness } \mathrm{x}]^{+}\right)\right]^{-} \text {. }
$$

We've presented our theory of facts. However, let's admit it: our clarification of negative facts is achieved through the notion of anti-instantiation. And what kind of clarification can that be, given that anti-instantiation is opaque? We admit the opacity. We deny that, consequently, no clarification has been achieved. The clarification is in linking negative facts to something any theory of substantial facts requires anyway: non-mereological composition. The link, we admit, is forged by postulating two basic kinds of non-mereological composition, and we have not said what the difference between them is. Clearly, there is a qualitative ontological cost. But ontological cost is not, in itself, a cogent argument against purchasing the postulate. We must also look at the work it does. This we do in the remainder of the paper, by showing how our theory accords with various forms of the Eleatic principle.

2I. Strictly speaking, we introduce a family of predicates ' $I \mathrm{n}^{\tau}$ ' for each type complex type $\tau$ (but not $\mathrm{f}$ or i). For the purposes of our discussion below, we can ignore the type superscript and assume that the correct predicate has been used to make a well-formed term. 


\section{The Eleatic Principle}

In this section, we discuss various criteria of existence which have been used to argue against negative facts. Molnar (2000) wields the Eleatic principle, according to which everything that exists has effects. The argument against negative facts is then:

\section{N egative facts are causally inert; they have no effects. Therefore, they do not exist.}

We shall not question the Eleatic principle, so understood, here. We focus on the premise that negatives are causally inert. Why believe this thesis? One thought is that negative facts cannot be causes because they are absences of being and consequently there is nothing there to do any causing. This argument is guilty of the confusion we pointed out in $\mathbb{S}_{3}$ : confusing that-which-is-absent (e.g., lakedwelling hippos) with the absence (the negative fact) itself. One might contend that causation is not the kind of relation that can hold between negative facts and other facts. But why not, given that negative facts exist in just the same sense as positive facts? One would need a special argument to conclude that only positive facts can enter into relations of causation.

Ordinary intuition seems to agree with our verdict: it does not shrink from using negative-talk in relation to causal claims. We are happy to assert that Bob's not watering the plants caused them to die. The enemies of causation by absence frequently contend that such claims are not cases of real causation. Dowe (200I) argues that the truth-conditions of such claims are captured in terms of counterfactuals: had Bob watered the plants, his doing so would have caused the plants to live. But two concerns arise here. First, it is not clear that such counterfactual paraphrasing works. We can imagine cases of preemption involving negative causation. Suppose that, had Bob watered the plants, an evil scientist would have sprayed poison on them, so that they would have died anyway. Still, we want to say that Bob's not watering the plants caused them to die. Secondly, even if the counterfactual paraphrase strategy worked, it would not provide a compelling argument that real causation takes only the positive form. It would show only that there are interesting counterfactual conditions mirroring causation by absence. It is only because the objectors are already convinced that negatives cannot cause that they think they must find some alternative account of causal commitments for the negative case. ${ }^{22}$

Dowe (2009) offers a further argument against causation by negatives, based on the work of Hall (2004). Dowe is not interested in wielding the Eleatic principle against negative facts but, as he denies that negative facts are causally efficacious, the would-be Eleaticist could use Dowe's argument. Dowe argues that if you let negatives cause, then you are going to violate the principle from Special Relativity

22. Perhaps they are impressed with the kind of process theory of causation offered by Dowe (2000), which cannot allow that negatives enter into causal relations. But Dowe's kind of process theory hardly provides the prospect of a general account of causation. A lot of positive causation, say in the social sciences, cannot be captured by a process theory. 
(STR) that no signals can travel faster than the speed of light. Dowe interprets that principle as entailing that all effects occur within the forward light cone of the cause. ${ }^{23}$ Dowe's argument depends on the premise that negative facts are located, which we accept $\left(\mathbb{S}_{3}\right)$. We said that a negative fact is located at the (possibly discontinuous) spatiotemporal region where its concrete constituents are located.

Now suppose that Bob's not watering his plants in Glebe, Sydney, caused their death at $\mathrm{t}^{*}$. Bob did not water the plants because he was kidnapped by Tralfamadorians, aliens who whisked Bob away, sequestering him in a zoo 2 million light years from earth. The four dimensional region that is the location of the fact that Bob did not water his plants is a discontinuous region, part of which is in Glebe and part of which is located 2 million light years away. It is true that the effect in Glebe, the plant's dying, is not in the forward light cone of Bob in Tralfamadore. But what's relevant to the STR principle is not just Bob's location at $t^{*}$, but rather the location of the fact that Bob did not water his plants at $t^{*}$. As this fact's location includes parts of Glebe, its forward light cone includes the effect. There is no violation of STR, as understood by Dowe.

We now turn to another way of wielding the Eleatic principle against negative facts. Why can't an opponent of negative facts refine the principle by replacing its reference to causation by reference to the transmission of momentum or energy from one spatial region to another? This is what Hall (2004) calls productive causation. A new argument against negative facts, using this refined version of the Eleatic principle, is:

\section{If putative facts cannot enter into relations of productive causation, then they do not exist. Negative facts do not enter into relations of productive causation. So they do not exist.}

What does productive causation require? It requires: (i) a physical process, understood as a purely positive set of atomic facts meeting certain conditions of spatiotemporal continuity; and (ii) certain nomic relations linking the facts entering the process. We note that nomic relations can link negative facts. A lack of a certain physical structure nomically entails the lack of certain kinds of events, such as spontaneous decay. So, negative facts can enter into nomic relations. The present Eleatic argument against negative facts, then, seems to be nothing more than this: negative facts cannot be linked to positive facts by certain kinds of nomic relations. This might be an interesting fact about negative facts but, even if the case, it is hardly an argument for their non-existence.

23. It is not obvious that we should accept Dowe's interpretation of the STR principle. Hall's (2004) paper, which inspires Dowe's argument, concludes that the kind of causation governing negatives, which Hall calls dependence causation, does not have to conform to the STR principle. Productive causation, which involves processes such as the transmission of momentum or energy from one spatial region to another, on the other hand, does conform to the STR principle. As ours is a case of dependence causation, it is unclear why Dowe thinks that the STR principle is relevant. 


\section{Constitution and Holes}

The Eleatic principle does not provide us with tools to wrench negative facts from the bosom of existence. There are arguments invoking cousins of the Eleatic principle, however, which offer the prospect of doing so. The Eleatic principle demands that existing things enter into causal relations. There is a kindred relation into which we could demand that existing things enter: constitution. Material objects, one might say, are somehow constituted through the facts about them. It is in virtue of thin particulars instantiating properties that we have thick particulars, the things we know and love: people, cats, MacBooks. This suggests that positive atomic facts are intimately bound up with the nature of material things, for they participate in the constitution of those things. This idea points to a criterion of existence: to be real is to be a potential constitutor. So let's assume that principle. Wielded against negative facts, one can then argue:

N egative facts have no role in the constitution of material things. But if they have no roles in constitution, they cannot exist. Therefore, they do not exist.

However, we feel obliged to point out that there are material objects which are constituted through negative facts.

Donuts are material objects. The thing about donuts, however, is that they have holes. If you get rid of the donut hole by filling it up with more donut-dough, then there is no longer a donut. But what are holes? According to Hoffman and Richards (I985), holes are mereological fusions of negative and positive parts. But that seems wrong, since the hole does not include any of the positive stuff that surrounds it. Another idea is that holes are hole-linings, i.e., the inner surfaces of the surrounding things (Lewis and Lewis 1970). But this view does not accord with the idea that holes can be filled up. Holes pose something of a philosophical quandary and, perhaps as a result of their mystery, are often treated as immaterial entities (Casati and Varzi I994). Yet we seem to be able to perceive holes, gaps, dents and the like. The view of holes as immaterial objects is, we think, very much in line with thinking of the negative as the metaphysically undead.

Given our acceptance of negative facts, we can offer a story about holes on which they are material entities. If there is a donut hole then there is a spatial region involving the instantiation of donut-dough which is intimately connected with an absence thereof. In $\rrbracket_{3}$, we identified absences with negative facts and so we propose to treat a hole as a negative fact surrounded, spatially, by an appropriate positive fact (recall that we treat facts as spatiotemporally located entities). More precisely: an absence of F-stuff (a negative fact) constitutes a hole insofar as it is surrounded by the presence of $F$-stuff (a positive fact). Let $O$ be the binary occupying relation which holds between a concrete particular and the spatial region it occupies. Then $\left[\ln \lambda \times\left([F x]^{+} \oplus[O \mathrm{xr}]^{+}\right)\right]^{-}$is the fact that no F-stuff occupies region $r$. This fact constitutes a hole when $r$ is spatially surrounded by F-stuff.

On this view, holes are very much material objects, for (i) a negative fact is a hole only insofar as it is appropriately related to surrounding material stuff; 
and (ii) there is no reason to view anti-instantiations of material properties by material things as non-material. We can't give a complete theory here, but we can say something informative. For example, you fill the hole with $\mathrm{G}$-stuff (without destroying the hole) by creating the positive fact $\left[\ln \lambda x\left([G x]^{+} \oplus[O x r]^{+}\right)\right]^{+}$and retaining $\left[\ln \lambda x\left([F x]^{+} \oplus[O x r]^{+}\right)\right]^{-}$(where Fness and Gness are distinct). You enlarge the hole by enlarging $r$ and retaining $\left[\operatorname{In} \lambda x\left([F x]^{+} \oplus[O x r]^{+}\right)\right]^{-}$and

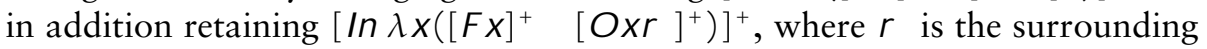
region. And so on. This theory can be extended to analyse gaps, dents and the like, by fine-tuning the account of regions in which negative facts are located and their relation to the surrounding positive facts.

One may object that it is a category error to identify holes with negative facts: aren't holes particulars, not facts? But on our view, negative facts are genuine entities. We can predicate things of facts, just as we can of holes, and (at least some) negative facts are spatially located, just as holes are. There is also independent positive evidence that negative facts can constitute holes, for negative-fact-talk can play the same role in explanations as hole-talk. How is it that the boys were able to spy on the girls? We can say: because of the hole in the wall. Or we can say: because of the fact that this small region of the wall wasn't filled in with brick. That talk of the appropriate negative fact may be substituted for talk of a hole in an explanation adds weight to our contention that the fact constitutes the hole. If holes are like this and holes exist, then the argument against negative facts as non-constitutors fails.

Should one really believe in holes? We think one should, though we cannot argue for that in detail here. Holes are explanatory, as in the example above. The basic concepts of topology depend on holes: solids without holes and solids with one or more holes represent a basic distinction in topology. Many physical processes involve holes. Osmosis, for example, is a process partly explained by holes in molecule-thick surfaces; the physical process is inextricably bound up with holes. Holes, therefore, enter into physical processes and, to that extent, so do negative facts. So the argument against negative facts as non-constitutors fails. Moreover, we now have a good reason to believe in negative facts: holes exist and are best understood as constituted by negative facts.

\section{Negative Facts and Perception}

The causal Eleatic principle and its more liberal cousin just examined, the constitution Eleatic principle, have not dislodged rational belief in negative facts, for we have good reason to believe that negative facts enter into relations of causation and constitution. But another kind of relational argument against negative facts beckons: concrete existence, it might be contended, requires some kind of in-principle perceptibility. All the basic constituents of a concrete thing need to be perceivable in principle (although, in cases such as the physical universe in its entirety, the whole may be imperceptible). The thought now is that negative facts cannot be perceived. Here is an argument:

Negative facts cannot be perceived. Therefore, they do not exist. 
We will not address the perceptibility criterion. Rather, let us address the charge that negative facts are imperceptible.

The evidence of ordinary intuition, at least in relation to perceptual reports, does not support this premise. We assert things like $X$ saw that $y$ was not $F$ all the time. The denier of negative-fact perceptibility could defend themselves here: these attributions really report the fact that $\mathrm{X}$ infers that $\mathrm{y}$ is not $\mathrm{F}$ on the basis of some perceptual experience of objects and positive facts. Fine! But so far we find no argument that negatives cannot be perceived. Furthermore, one should doubt, given the analysis of negative facts that we defend, that negative facts should be any less perceivable than positive facts. As we noted in the case of causation, an atomic fact of the form $[\mathrm{Fa}]^{+}$and its negative counterpart $[\mathrm{Fa}]^{-}$have the same constituents: $\mathrm{a}$ and $\mathrm{F}$. Why think that one kind of non-mereological composition is perceptible whereas the other is not?

Here are two lines of thought which might articulate that idea. Perception, one might contend, depends on looks. It is things looking a certain way that enables us to perceive them. But the negative facts that the lake is not purple and that the lake is not yellow look the same. There is no particular kind of look associated with these facts. Consider then the argument:

Perception must be based on looks. Negative facts have no look. Therefore, negative facts cannot be perceived and so cannot exist.

Our concern is that the argument confuses the logic of looks. It is people, cats and MacBooks that look a certain way, not facts. A chair can look blue, whereas the fact that the chair is blue is not the kind of thing that can look blue. Facts about material things, be they positive or negative, do not look any particular way. If so, the present line of argumentation could be used to conclude that no kind of fact is perceivable, which is surely too strong 
the analogous case of computer vision, in which an edge is defined as a sharp change in intensity (i.e. in brightness of the pixels in a digital image). Computerized edge-detection locates such intensity transitions (Basu 2002, 252). ${ }^{24}$ One might think, therefore, that an image can be represented as positive information only (as an array of pixel intensities) and so that edge detection algorithms make no appeal to negative information. This is not the case, however. The value representing the intensity of each pixel contains both positive and negative information: a pixel's intensity value says that the pixel is at least this bright but no brighter. The numerical value associated with each pixel encodes both a positive and a negative fact. We suspect that similar story holds of edge-detection in human perception.

That one must perceive negative facts to detect edges is not surprising. After all, an edge is simply a qualitative presence related to a qualitative absence. In this regard, edges are like holes $\left(\mathbb{S}_{7}\right)$ in reverse. They are constituted by a positive fact insofar as it is spatially juxtaposed to a negative fact. Edges, just like holes, cannot be understood without appeal to negative as well as positive facts. But clearly, edges can be perceived, just as holes can. To do so, one needs to perceive both the positive and the negative fact. So qualitative character sometimes depends on absences and hence on negative facts, and we must reject premise (ii). As a consequence, we do not see that there is any argument from imperceptibility against negative facts.

\section{Putting Negative Facts to Use}

We have argued that the case against negative facts, correctly understood, is rather weak. We will now argue that the case in their favour is strong, for it is our contention that negative facts play a number of making-roles. In $\$ 6$ above, we argued that negative facts play a role in causation (or 'event-making'). The fact that Bob did not water his plants is causally responsible for their death a few days later. Then, in $\$ 7$, we argued that negative facts constitute holes (and related phenomena), insofar as they are appropriately related to (e.g., being surrounded by) positive facts. So negative facts can play roles in event-making and in constituting, or making up, objects.

In addition, negative facts play roles in chance-making and truth-making, as we shall argue in the remainder of this section. First, let's consider chances. We hold that chances are real and, as single case objective probabilities, features of the local world. Chances, as well as truths, require grounding in the world. Suppose a box contains an isotope I with a certain half-life such that, at $t$, there is a 0.6 chance of it decaying within 5 minutes, assuming that the isotope is not destroyed within that time period. That chance is not an ontologically primitive feature of time t. Our concern is with what features of the world explain this chance. Grounding and explanation are asymmetric relations: the ways the world is (the facts) ground and explain the chances things have and the truths there are, but

24. In an 8-bit grayscale image, for example, the intensity of each pixel is stored as an integer between $\circ$ and 255 . For a survey of edge detection techniques in artificial intelligence, see Basu 2002. 
neither those chances nor those truths ground or explain the ways the world is. So one cannot appeal to supervenience, which is a symmetric relation, to ground or explanation chances (or truths). Instead, an explanation of the fact that I at $t$ has a 0.6 chance of decaying must contain the following information:

(a) I has physical structure $\mathbf{X}$; and

(b) there are no isotope destroyers or heavy particles moving towards I within its reverse light cone, 5 minutes after $t$.

This explanation invokes negativity: (b) makes apparently ineliminable reference to negative facts. The explanation here links facts in grounding relations. We take this to show that negative facts qua constituents of the world are required for a full account of chance-making.

Now let's consider truth-making, a subject about which much of the current theorising about facts is concerned. We can distinguish two broad positions on how negative truths (such as true negative existentials) come to be true.

N on-maximalism: some truths (the 'positive' truths) are made true by some entity, but some (the 'negative' truths) are not made true by any entity.

Maximalism: all truths are made true by some entity or other. ${ }^{25}$

We don't have space here to give a full argument against non-maximalism here. ${ }^{26}$ But in brief: we suspect non-maximalism of supporting a dualistic theory of truth, a making-theory for positive truths and some other theory for the rest. But if that latter theory is a theory of truth at all, why not apply it across the board and do away with truthmaking even for positive truths? By contrast, if one thinks (as Cameron (2007) and Rodriguez-Pereyra (2005) do) that truth requires grounding, then there can be no ungrounded truths. Truth-making is a form of grounding relation and relations relate existing entities (Rodriguez-Pereyra 2005). If so, all truths require something in the world to make them true. The principle problem for maximalism is: what kind of entity makes the negative truths true? We have already given our answer $\left(\mathbb{S}_{3}\right.$ and $\mathbb{S}_{5}$ ): negative facts (including negative existential facts and general facts) do the making in such cases.

Of the various making-roles that negative facts can play, truth-making is perhaps the most frequently discussed. One of our aims has been to redress this imbalance by highlighting other making-roles: constitution (e.g., hole-making), causation (event-making) and chance-making. Nevertheless, we think that the argument from truth-making is a perfectly good reason for believing in negative facts; our point here is that it is but one amongst several such reasons. So here is a form of the Eleatic principle of which we approve: material things exist if and only if they can enter into making relations. Negative facts enter into making relations. So they exist.

25. Melia (2005) holds a third (nominalist) view, on which truths are made true but not by existing entities. We do not see how a truth can be made true (i.e. grounded) unless it is made true (grounded) by something that exists.

26. See Jago 20I0a for one of the present author's arguments against non-maximalism. 


\section{Io The Undead Put to Rest}

What lies behind the feeling that there cannot be negative facts, noted by Russell (I985): the innate horror, up there with fear of snakes and spiders, which attends contemplation of the negative? We think it's the vampiric image of negative facts as amongst the metaphysically undead: non-existence somehow masquerading as existence. But that idea, as we have shown, is not really part of any serious conception of negative facts. We have argued not only that negative facts are a coherent idea, but also that there are no cogent arguments against their existence. There are, moreover, many good reasons to want them around. Negative facts play irreducible making-roles. We accept the principle that making is the mark of existence. Negative facts make. So they exist.

Words: $8,47 \mathrm{I}$ (including footnotes, abstract $\&$ bibliography)

\section{References}

Armstrong, D. (1997). A World of States of Affairs, Cambridge University Press, Cambridge.

Barendregt, H. (1984). The Lambda Calculus: its Syntax and Semantics, North Holland.

Barendregt, H. (I992). Lambda calculi with types, Handbook of Logic in Computer Science, Vol. 2, Oxford University Press, Oxford, pp. I I 7-309.

Basu, M. (2002). Gaussian-based edge-detection methods: a survey, IEEE Trans. Systems, Man, and Cybernetics, Part C: Applications and Rev. 32: 252-26o.

Beall, J. (2000). On truthmakers for negative truths, Australian Journal of Philosophy $78(2): 264-268$.

Cameron, R. (2007). How to be a truthmaker maximalist, Nous 42(3): 4IO-42I.

Casati, R. and Varzi, A. (1994). Holes and other Superf cialities, MIT Press, Cambridge, MA.

Dowe, P. (2000). Physical Causation, Cambridge University Press, Cambridge.

Dowe, P. (200I). A counterfactual theory of prevention and 'causation' by omission, Australian Journal of Philosophy 59: 216-226.

Dowe, P. (2009). Absences, possible causation, and the problem of non-locality, TheMonist 92: 24-4I.

Fine, K. (1982). First-order modal theories III-facts, Synthese 53(I): 43-I 22.

Greenough, P. (2008). Indeterminate truth, Midwest Studies In Philosophy 32(I): 2I3-24I.

Hall, N. (2004). Two concepts of causation, in J. Collins, N. Hall and L. Paul (eds), Causation and Counterfactuals, MIT Press, Cambridge, MA, pp. I 8 I-204.

Hoffman, D. and Richards, W. (I985). Parts of recognition, Cognition s 8: 65-96.

Jago, M. (20Iоa). The non-maximalist's dilemma. Draft MS. 
Jago, M. (20Iob). Setting the facts straight. Draft MS.

Lewis, D. (1983). New work for a theory of universals, Australian Journal of Philosophy 6I: $347-77$.

Lewis, D. and Lewis, S. (I970). Holes, Australasian Journal of Philosophy 48(2): 206-2 I 2.

Melia, J. (2005). Truthmaking without truthmakers, in H. Beebee and J. Dodd (eds), Truthmakers: the contemporary debate, Oxford University Press, Oxford, pp. 67-84.

Molnar, G. (2000). Truthmakers for negative truths, Australasian Journal of Philosophy $78(\mathrm{I}): 72-86$.

Mumford, S. (2007). Negative truth and falsehood, Proceedings of the Aristotelian Society IO7(I): 45-7I.

Neale, S. (200I). Facing Facts, Oxford University Press, Oxford.

Parsons, J. (2006). Negative truths from positive facts?, Australasian Journal of Philosophy $84(4): 59 I-602$.

Restall, G. (2004). One way to face facts, The Philosophical Q uarterly 54(216): 420-426.

Rodriguez-Pereyra, G. (2005). Why truthmakers, in J. Dodd and H. Beebee (eds), Truthmakers, Oxford University Press, pp. I7-3 I.

Russell, B. (I985). The philosophy of logical atomism, in D. Pears (ed.), ThePhilosophy of Logical Atomism, Open Court, La Salle, pp. 35-I 55.

Sorensen, R. (2004). Vagueness and Contradiction, Oxford University Press, Oxford.

van Fraassen, B. (1969). Facts and tautological entailments, Journal of Philosophy 66(I 5 ): 477-487.

Zalta, E. (I993). Twenty-five basic theorems in situation and world theory, Journal of Philosophical Logic 22(4): $385-428$. 